\title{
Residue Dynamics of Phoxim in Pericarp, Sarcocarp and Kernel of Apple
}

\author{
Yunxia Luan ${ }^{1,2}$, Hua Ping ${ }^{1,2}$, and Ligang Pan ${ }^{1,2, *}$ \\ ${ }^{1}$ Beijing Research Center for Agrifood Testing and Farmland Monitoring, \\ 100097 Beijing, China \\ ${ }^{2}$ Beijing Research Center for Information Technology in Agriculture, \\ 100097 Beijing, China \\ Tel.: +8610 51503031; Fax: +86 1051503406 \\ panlg@nercita.org.cn, luanyx@nercita.org.cn
}

\begin{abstract}
The temporal and spatial variations of phoxim residue in apples were studied. The insecticide phoxim was applied by spraying according to the recommended dosage. The phoxim residues in different parts of apple were extracted with acetonitrile, purified by primary secondary amine (PSA) and C18 filler, and then determined by gel permeation chromatography-gas chromatograph mass spectrometer (GPC-GC/MS). It was disclosed that the half-life of phoxim in apples was 1.64 days. The contents of the phoxim residue in different parts of apple could be ranked as follows : pericarp > entire apple > sarcocarp $>$ kernel, and the ratio of residue in pericarp and the entire apple ranged from 6.241 to 9.262 in groups with different specific surface area. There were significant differences in the contents of residues between the pericarp and entire apple, which provided the evidence in the theory instruction and design basis to the sampling method of pesticide residue based on pericarp.
\end{abstract}

Keywords: Apple; Phoxim; Sampling method; Residue dynamics; GPCGC/MS.

\section{Introduction}

Agricultural product quality and safety has been given higher requirements in recent years. Currently, the issues caused by excessive pesticide residues in edible agricultural products attracted the social attention to the safety and monitoring of vegetable production processes. Pesticides are used to control insects, weeds, and disease throughout the world [1]. Although their use leads to increased crop yields and improvements to the food quality, pesticide residue levels in food are of increasing concern to the public. Due to the abuse and misuse of pesticide, pesticide residues severely influence people's health. Organophosphorus (OP) pesticides are the most widely used agricultural pesticide [2]. More than $70 \%$ of that are hyper-toxic or high-toxic, and normally are forbidden to use in planting fruits and vegetables [3].

OP insecticides are routinely applied to fruit crops (e.g., apple, peach, and pear) that are subject to consumption as single servings [4, 5]. Consumption of individual

\footnotetext{
* Corresponding author.
} 
fruits containing high OP insecticide residues may result in high exposure over a short period of time. Residue testing for the presence of pesticides, however, is routinely performed on composites of 5 to 10 individual or unit samples rather than samples of individual fruit [6-8]. The use of data derived from composite samples may result in the underestimation of residues present in individual fruit because dilution may occur during compositing and, therefore, may not accurately represent exposure in single serving commodities $[9,10]$.

Variability factors have been established to indicate how much residue concentrations measured in individual samples vary from levels observed in composite samples [11-13]. Variability factors have been calculated by dividing the maximum pesticide concentration observed in an individual or unit sample by the mean composite level [13]. In recent years, studies to establish residue levels observed in individual samples relative to composite samples have been performed in a variety of crops (e.g., apples, carrots, oranges, potatoes, and tomatoes) [14-17]. Residue data in unit samples of individual commodities are required to establish variability factors for comparison with default values currently being used. Recently, variability also has been estimated using the 97.5th percentile concentration data for individual samples, rather than maximum levels, where sufficient data exist. Sampling and sample preparation are critical steps in analysis, and cannot be treated separately from sample treatment; rather the processes must be conducted together. However, limited data have been reported that directly compare the sampling procedures against one another. Even less information is published regarding the most appropriate part of the material to use in conjunction with a sampling procedure for pesticides detection, which is particularly important to the portable rapid detector of contamination in fruits and vegetables.

The present study addressed how well pesticide residue levels measured in composite samples represent concentrations in different parts of apples, in order to improve sampling method of single serving foods. A within-laboratory trial was performed to ensure that insecticides were applied at known rates, times, and following label practices. By sampling from the selected apples, individual apples were not mixed with others during spray and distribution. The temporal and spatial variations of phoxim residue in apples could be established.

\section{Materials and Methods}

\subsection{Chemicals and Reagents}

Phoxim standard was purchased from National Institute of Metrology (China). The dispersive solid phase extraction sorbents of primary secondary amine (PSA) and C18 were purchased from Agela Technologies Inc. High-purity solvents cyclohexane, acetone, and acetonitrile were all purchased from Fisher Chemicals (Pittsburg, PA, USA). Analytical reagents magnesium sulfate $\left(\mathrm{MgSO}_{4}\right)$ and sodium chloride $(\mathrm{NaCl})$ were purchased from Beijing Chemical Reagents Company (China).

\subsection{Apparatus}

The gel permeation chromatography (GPC) equipped with gas chromatography mass spectrometry system (GC/MS) was used. The GPC consists of two LC-10ADvp 
pumps, a SIL-10ADvp auto-sampler, a Shodex CLNpak EV-200AC column (2mm i.d. $\times 150 \mathrm{~mm}$ ) and CTO-10ASvp column oven, a SPD-10Avp UV detector, two FCV$12 \mathrm{AH}$ flow channel selection valves (RV.A, RV.B) and a SCL 10Avp system controller. GC/MS machine is a Shimadzu GC/MS-QP2010 instrumentation equipped with a PTV-2010 large-volume injection device.

GC/MS data analysis was triggered by a contact closure start signal from the HPLC controller. Data acquisition was performed using a C-R8A plus data processor. All these parts are the products of Shimadzu (Kyoto, Japan), except the Shodex CLNpak EV-200AC column (Shoko Co., Tokyo, Japan). Acetone/ cyclohexane mixing solvent $(3 / 7, \mathrm{v} / \mathrm{v})$ was used as the mobile phase of GPC, and the flow rate was set at $0.1 \mathrm{ml} / \mathrm{min}$. The mobile phase was degassed using DGU-14A degasser (Shimadzu), and the GPC column was kept at $40^{\circ} \mathrm{C}$ in the column oven.

\subsection{Sample Preparation}

All samples were purchased at local markets in Beijing. Samples were extensively collected to achieve good sample homogeneity. Phoxim was applied using an air blast sprayer and application rates guaranteed to contain 50\% phoxim emulsifiable with the dilution multiple of 1:500. Apples were cored and sliced into 5 segments [pericarp(P), sarcocarp1(S1,S2,S3,S4) and kernel] separately, using a corer/slicer retailed and peeling machine for domestic use. The first and alternate slices of each apple were taken, chopped manually using a knife, and placed in a plastic bag for storage at $-25^{\circ} \mathrm{C}$ until extraction and analysis. The remaining segments of individual apples were retained in separate bags for preparation of composite samples. Composites were constructed from the apples prepared from each group by randomly selecting 8 apples for each treat, regardless of the style and weight of each apple. Composite samples were prepared by thoroughly mixing the chopped apple pieces from the bags containing the retained portions of the individual apples. Composite samples were frozen until extraction and analysis. To prepare each sample, $10 \mathrm{~g}$ of a previously homogenized food material was transferred into a suitable glass vessel. Then, $10 \mathrm{ml}$ acetonitrile was added to each sample using an adjustable-volume solvent dispenser. The glass vessels were capped before vortex mixing for 1 min at maximum speed. Once the initial sample mixing was completed, $1 \mathrm{~g} \mathrm{NaCl}$ and $4 \mathrm{~g}$ anhydrous $\mathrm{MgSO}_{4}$ were added and mixed immediately on a Vortex mixer for $1 \mathrm{~min}$. It was important to note that this step must be taken immediately after the initial mixing step to prevent the formation of $\mathrm{MgSO}_{4}$ conglomerates. To separate phases, samples were centrifuged for $10 \mathrm{~min}$ at $1570 \times \mathrm{g}$. Using an adjustable repeating pipette, $1.0 \mathrm{ml}$ aliquot of upper acetonitrile layer was transferred into a $1.5 \mathrm{ml}$ flip-top microcentrifuge vial containing $150 \mathrm{mg}$ anhydrous $\mathrm{MgSO}_{4}$ and $50 \mathrm{mg}$ PSA sorbent. The vial was tightly capped and shaken on a vortex mixer for $1 \mathrm{~min}$ before extraction. Then the mixed extraction solution was centrifuged for $5 \mathrm{~min}$ to separate solids from solution. The solution was filtered through a $0.45 \mu \mathrm{m}$ syringe filter for GPC-GC/MS analysis.

\subsection{Determination of Phoxim in Apple by GPC-GC/MS}

Analysis was performed by GPC-GC/MS. The analytical GC/MS equipped with a PTV-2010 large-volume injection device was carried out using deactivated silica 
tubing $(5 \mathrm{~m} \times 0.53 \mathrm{~mm}$ i.d.) , a Rtx-5MS pre-column $(5 \mathrm{~m} \times 0.25 \mathrm{~mm})$ and a Rtx-5MS column $(30 \mathrm{~m} \times 0.25 \mathrm{~mm} \times 0.25 \mu \mathrm{m}$ film thickness; coated with $5 \%$ phenyl and $95 \%$ methylpolysiloxane; Restek Corporation, Bellefonte, Palo Alto, USA). The temperature of the PTV injector was set at $120^{\circ} \mathrm{C}$ for the initial $5 \mathrm{~min}$ of sampling time, and then increased to $250^{\circ} \mathrm{C}$ at $100^{\circ} \mathrm{C} / \mathrm{min}$. The oven temperature was maintained at $80^{\circ} \mathrm{C}$ for $5 \mathrm{~min}$, subsequently increased to $280^{\circ} \mathrm{C}$ at a rate of $8^{\circ} \mathrm{C} / \mathrm{min}$, and then held constant for $10 \mathrm{~min}$. The quadrupole mass spectrometer was operated in the electron impact ion (EI) mode. Ion source temperature and interface temperature were set at $200^{\circ} \mathrm{C}$ and $250^{\circ} \mathrm{C}$, respectively. The mass spectrometer was operated in an ionizing energy of $70 \mathrm{eV}$. Injection volumes were $1 \mu \mathrm{l}$ for all analyses. Helium (99.999\%) was used as the carrier gas.

\subsection{Quality Assurance/Quality Control}

Apples from market without phoxim pesticide were obtained, which were prepared the same as the rinsed and peeled apples, and used to prepare blank apple matrix for quality assurance testing. With each set of samples (8-10 samples per set) extracted and analyzed, two aliquots of blank apple matrix were prepared for separate extraction followed by extraction, cleanup, and analysis as for all other samples. Background levels of some analyses were periodically detected in the blank matrix samples and were used for background subtraction in the determination of recovery from spiked matrix only; residue concentrations in samples were not blank corrected. No traces of the phoxim were observed in any of the analyzed reagent blanks.

\section{Results and Discussion}

\subsection{Degradation Dynamics of Phoxim in Apple}

The phoxim pesticide was found to be beyond the detection limit in all the samples analyzed in the present study. In general, phoxim was found at elevated levels in entire and different parts of apples prepared from treatment relative to those control without sprayed by phoxim. Concentrations of phoxim were detected from the day applied to 28 days after treatment (Fig. 1). The degradation rate in the three days after treatment was $24.36 \%, 48.73 \%$ and $54.18 \%$, respectively. The rate of degradation changed slowly between the 5th day to the 10th day, while the concentration of phoxim suddenly dropped after 11 days.

Phoxim residues were mainly found in pericarp and the outer laying sarcocarp. However, low levels of phoxim was also detected in the interior sarcocarp and kernel. Residue levels ranged from $0.0057 \mathrm{mg} / \mathrm{kg}$ to $0.1100 \mathrm{mg} / \mathrm{kg}$ in the entire apples, $0.0061 \mathrm{mg} / \mathrm{kg}$ to $0.4469 \mathrm{mg} / \mathrm{kg}$ in pericarp and reduced level in sarcocarp adjacent to pericarp (Tab. 1). Phoxim was not detected in any solvent blanks that were processed along with samples, indicating that background levels of this compound were not due to lab contamination.

Successive decline was observed during storage period (Fig. 1). It should be noted that the relative increase level of phoxim in sarcocarp and kernel after applied 10 days earlier (Tab.1). In apples analyzed after 28 days treatment, no residues were found in 
sarcocarp and kernel any more. It was assumed that the reduction of phoxim during the storage might be caused by its relatively high persistence to hydrolysis, and that the residues detected in kernel might be related to extensive vascular system around kernel.

Table 1. Ratio of residue in pericarp and the entire apple in apples with different SSA

\begin{tabular}{lllll}
\hline \multirow{2}{*}{$\begin{array}{l}\text { Days after } \\
\text { treatment }\end{array}$} & Total apple & pericarp & sarcocarp & kernel \\
\hline 0 & 0.1100 & 0.4469 & 0.0013 & ND \\
1 & 0.0832 & 0.3644 & 0.0015 & 0.0009 \\
2 & 0.0564 & 0.3572 & 0.0019 & 0.0011 \\
3 & 0.0504 & 0.3250 & 0.0025 & ND \\
5 & 0.0485 & 0.3071 & 0.0013 & 0.0008 \\
7 & 0.0449 & 0.3057 & 0.0033 & 0.0009 \\
9 & 0.0410 & 0.2810 & 0.0030 & ND \\
11 & 0.0391 & 0.2567 & 0.0012 & ND \\
13 & 0.0164 & 0.1173 & 0.0009 & ND \\
15 & 0.0087 & 0.0433 & 0.0008 & ND \\
20 & 0.0069 & 0.0219 & 0.0006 & ND \\
28 & 0.0057 & 0.0061 & ND & ND \\
\hline
\end{tabular}

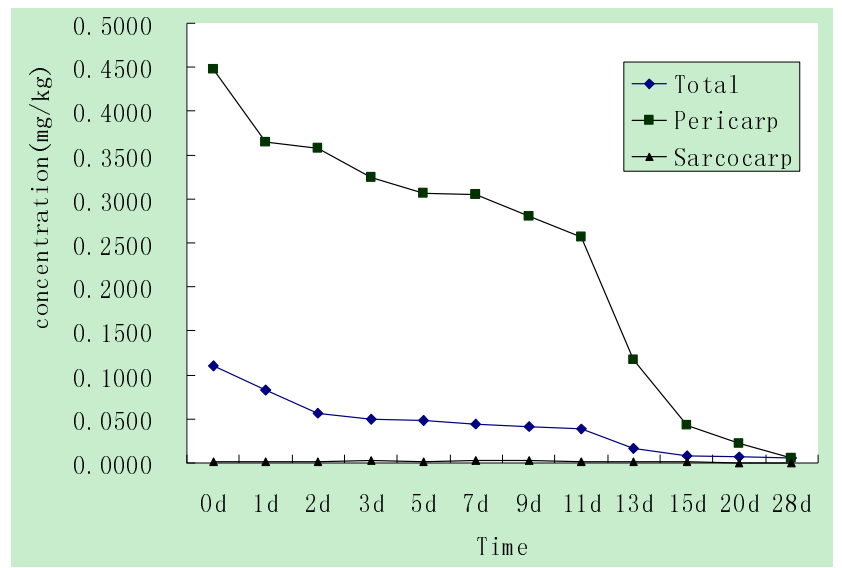

Fig. 1. Degradation of phoxime in different parts of apple

\subsection{Analysis of Phoxim in Different Parts of Apple}

In order to investigate the phoxim level in different parts of apples, segments from different layer of pericarp (P), sarcocarp (S1, S2, S3, S4) and kernel of individual 
apples were separated by using the peeling machine to avoid errors caused by manual operation (Fig.2). The residues observed in samples collected from different part of apples were shown in Figure 3.

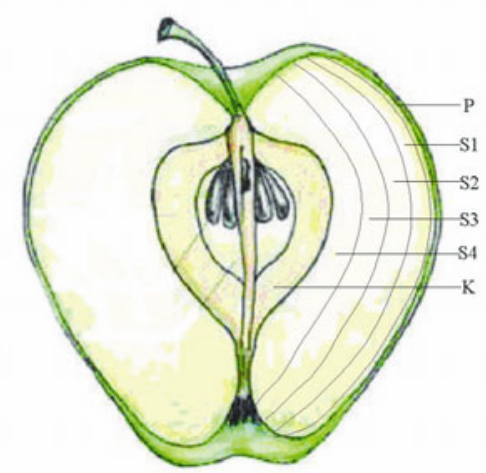

Fig. 2. Different sampling parts of apple P: pericarp of apples, S1: sarcocarp close to pericarp, S2: the second layer of sarcocarp, S3: the third of sarcocarp, S4: the sarcocarp near kernel, K: kernel or core of apples

The concentration of the total apple and pericarp was consistent with the result above with the crude sampling method. The maximum phoxim concentration was observed in pericarp. The phoxim residue order in different positions of apple was: pericarp $>$ total apple $>$ the sarcocarp close to pericarp $(\mathrm{S} 1)>$ kernel $>$ the sarcocarp near kernel (S4) (Fig. 3). Contrary to expectations, no phoxim residue was detected in S2 and S3, which was the main edible part of apples. Therefore, 85\% of the pesticide residues existed on the surface of the apple, and the degradation rate of pesticide residues in the entire apple was consistent with pericarp.

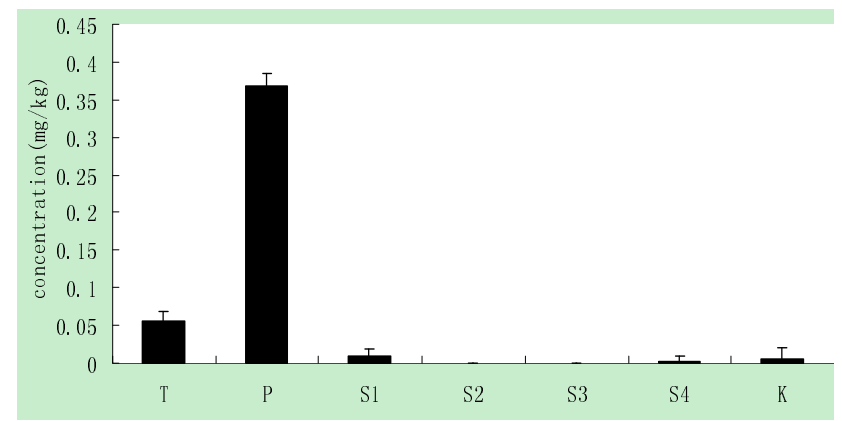

Fig. 3. Residues of phoxime in apple 6 days after treatment

\subsection{Relationship between Residues and Specific Surface Area}

Specific surface area (SSA) is a material property of solids which measures the total surface area per unit of mass, solid or bulk volume, or cross-sectional area. It is a 
derived scientific value that can be used to determine the type and properties of a material. It is defined either by surface area divided by mass (with units of $\mathrm{m}^{2} / \mathrm{kg}$ ).

The most representative sampling part of apple was the pericarp when phoxim applied by spray. Sample of pericarp instead of the whole homogenate would be efficient in pesticide residues analysis. Therefore, the ratio of residue in pericarp and the entire apple in apples was investigated in Fuji apples with different shapes and specific surface areas (Tab.2). Apples with higher weight had lower SSA, while the pesticide residue in pericarp was higher than the apples with lower SSA. The ratio of phoxim in pericarp and the entire apple ranged from 6.241 to 9.262 in groups with different SSA.

Table 2. Ratio of residue in pericarp and the entire apple in apples with different SSA Weight

\begin{tabular}{cccc}
\hline $\begin{array}{c}\text { Weight } \\
(\mathbf{g})\end{array}$ & $\begin{array}{c}\text { Surface Area } \\
\left(\mathbf{c m}^{2}\right)\end{array}$ & $\begin{array}{c}\text { SSA } \\
\left(\mathbf{c m}^{2} / \mathbf{g}\right)\end{array}$ & $\mathbf{C p / C t}$ \\
\hline $169.605 \pm 4.666$ & $170.750 \pm 6.583$ & 1.007 & 6.241 \\
$189.425 \pm 4.748$ & $180.093 \pm 2.512$ & 0.950 & 7.963 \\
$209.032 \pm 4.015$ & $190.377 \pm 2.926$ & 0.911 & 9.262 \\
\hline
\end{tabular}

\subsection{Discussion}

The problem of pesticide residue has become a chief obstacle to the apply industry internationalization in the world. At present, samples tested for the pesticide residue stem mainly from the entire apple, because the results from the sample have been generalized. However, pesticide residue of apples mainly exists in the periearp, a large number of pulp mixed into analysis sample would dilute the pesticide residues in pericarp, which would reduce the accuracy of detection, especially in the rapid detection. Therefore, the careful research to pesticide residue of apple periearp provides the theory instruction and the design basis to the reasonable and efficient sampling method.

Acknowledgments. This work was supported by the public foundation from Beijing Municipal Rural Affair Committee (20080901), National High Technology Research and Development Program 863 (2010AA10Z403, 2007AA10Z202) and Beijing Municipal Science and Technology Commission Program (Z09090501040901).

\section{References}

1. Wen, Y.X., Li, J.K., Zhang, X.M., Xu, J.: Studies on sensitivity and detection limit of phytoesterase on organophosphate pesticides. Food Science 27(09), 186-188 (2006); Mith, T.F., Waterman, M.S.: Identification of Common Molecular Subsequences. J. Mol. Biol. 147, 195-197 (1981)

2. Jensen, A.F., Petersen, A., Granby, K.: Cumulative risk assessment of the intake of organophosphorus and carbamate pesticides in the Danish diet. Food Addit. Contam. 20, 776-785 (2003) 
3. Mol, H.G.J., van Dam, R.C.J., Steijger, M.: Determination of polar organophosphorus pesticides in vegetables and fruits using liquid chromatography with tandem mass spectrometry: selection of extraction solvent. J. Chromatogr. A 1015, 119-127 (2003)

4. Zhou, H.Y., Gao, Z.X., Sun, S.M., Wang, H.Y.: Development and application of on-site fast detection techniques for food safety. Journal of Instrumental Analysis 27(7), 788-794 (2008)

5. Souza, M.S., de Potas, G.M., de D’Angelo, A.M.P.: Organophosphorous and organochlorine pesticides affect human placental phosphoinositides metabolism and PI-4 kinase activity. J. Biochem. Mol. Toxicol. 18, 30-36 (2004)

6. Giddings, J.M., Biever, R.C., Racke, K.D.: Fate of chlorpyrifos in outdoor pond microcosms and effects on growth and survival of bluegill sunfish. EnViron. Toxicol. Chem. 16, 2353-2362 (1997)

7. Andersson, A.: Comparison of pesticide residues in composite samples and in individual units: the Swedish approach to sampling. Food Addit. Contam. 17, 547-550 (2000)

8. Harris, C.A.: How the variability issue was uncovered: the history of the UK residue variability findings. Food Addit. Contam. 17, 491-495 (2000)

9. Pennycook, F.R., Diamand, E.M., Watterson, A., Howard, C.V.: Modeling of the dietary pesticide exposures of young children. Int. J. Occup. EnViron. Health 10, 304-309 (2004)

10. WHO (World Health Organization): Joint FAO/WHO consultation on food consumption and exposure assessment to chemicals in food, Geneva, Switzerland (February 10-14, 1997)

11. Suhre, F.B.: Pesticide residues and acute risk assessmentsthe US EPA approach. Food Addit. Contam. 17, 569-573 (2000)

12. Fernández-Cruz, M.L., Villarroya, M., Llanos, S., Alonso-Prados, J.L., Garcia-Baudin, J.M.: Field-incurred fenitirothion residues in kakis: comparison of individual fruits, composite samples, and peeled and cooked fruits. J. Agric. Food Chem. 52, 586-863 (2004)

13. Ambrus, A.: Within and between field variability of residue data and sampling implications. Food Addit. Contam. 17, 519-537 (2000)

14. Boulaid, M., Aguilera, A., Camacho, F., Soussi, M., Valverde, A.: Effect of household processing and unit-to-unit variability of pyrifenox, pyridaben, and tralomethrin residues in tomatoes. J. Agric. Food Chem. 53, 4054-4058 (2005)

15. Ambrus, A., Soboleva, E.: Contribution of sampling to the variability of pesticide residue data. J. AOAC Int. 87, 1368-1379 (2004)

16. Lentza-Rizos, C., Tsioumplekou, M.: Residues of aldicarb in oranges: a unit-to-unit variability study. Food Addit. Contam. 18, 886-897 (2001)

17. Lentza-Rizos, C., Balokas, A.: Residue levels of chlorpropham in individual tubers and composite samples of postharvest-treated potatoes. J. Agric. Food Chem. 49, 710-714 (2001) 